\title{
Magnetic Orientation of Microcylinders in Liquid Crystalline Diblock Copolymer and Clarification of Its Orientation Mechanism
}

\author{
Mitsu-aki ADACHI, Fumihiro TakaZawa, Naoki Tomikawa, \\ Masatoshi TOKITA, and Junji WATANABE ${ }^{\dagger}$ \\ Department of Organic and Polymeric Materials, Tokyo Institute of Technology, \\ Ookayama, Meguro-ku, Tokyo 152-8552, Japan
}

(Received August 24, 2006; Accepted November 8, 2006; Published December 14, 2006)

\begin{abstract}
The liquid crystal (LC) block copolymers composed of side-chain LC polymer (LCP) and polystyrene (PS) segments with various molecular weights and block contents were prepared by atom transfer radical polymerization, and their microsegregated structures were examined for magnetic alignment. Well-aligned samples have been prepared only for the low molecular weight block copolymers with cylindrical microdomains of PS dispersed in the LCP matrix. The prerequisites for perfect alignment of the microdomain structure are (1) coincidence of the disorder-order transition of the microdomain with the isotropic to nematic transition of the LCP segment, and (2) anchoring of the LC mesogen parallel to the microdomain interface. Well-oriented cylindrical and/or anisotropic microsegregated structures are also observed for the mixtures of a LC homopolymer and a LC block copolymer irrespective of extremely low PS segment content of 12-2\%. Such persistent formation of anisotropic PS microdomains in the LC field indicates a significant interplay between the microdomain and liquid crystalline structures. [doi:10.1295/polymj.PJ2006102]

KEY WORDS Magnetic Orientation / Liquid Crystalline Block Copolymer / Cylinder Microdomain / Side-Chain Liquid Crystalline Polymer /
\end{abstract}

Block copolymers have attracted attention because they demonstrate great promise as materials for application in various macroscopic and microscopic technologies. They can form the lamellae, hexagonalpacked cylinders, cubic-packed spheres and bicontinuous cubic gyroid structures. These microstructures need to be aligned well all over a mass for application, but it has been hardly achieved.

From this aspect, the liquid crystal polymer (LCP) is attractive since it can be easily oriented under external fields, such as mechanical, electric and magnetic fields. Among them, magnetic orientation is most interesting because it can provide higher degree of freedom for the shape of samples than mechanical orientation and no danger such as the dielectric breakdown encountered in electrical orientation. Magnetic field orients LCPs safely to provide a sample oriented well and uniformly over all the regions regardless of its macroscopic shape. In the side-chain type of LCPs, in fact, there are a number of achievements of the macroscopic orientation of polymeric structures with an application of a magnetic field during cooling from isotropic to LC phase. ${ }^{1-3}$

Here, we note an interplay between liquid crystallinity and microphase segregation of LC-coil diblock copolymers (LCBCP). ${ }^{4-11}$ Since the interface of microsegregation is able to act as an anchoring surface for liquid crystal orientation, the magnetic alignment of the liquid crystal segments can result in the orientation of microphase structure. According to this expectation, more recently, several approaches for the magnetic alignment of microdomain structure in LCBCP have been reported. ${ }^{10,11}$ Hamley et al. ${ }^{10}$ evaluated the orientation of microdomain structure in the smectic LC field of LCP segments with chiral component at the end of mesogenic moieties. They succeeded in orientating the cylindrical microdomain of LCP segment in polystyrene (PS) matrix (LCP-Cyl.) perpendicular to the magnetic field, and the orientational order of the microdomain was not high. They did not succeed in the orientation of the inverse morphology, i.e., PS cylinder in the LCP matrix (PS-Cyl.). On the other hand, Osuji et al. ${ }^{11}$ carried out an alignment of PS-Cyl. microdomain for LCBCP sample with a ferroelectric LCP segment. In this case, the uniaxial orientation was observed within minute grains of not more than $400 \mu \mathrm{m}$ in the sample because the negative diamagnetic anisotropy of the mesophase is essentially unsuitable for uniaxial orientation. The first successful preparation of perfectly aligned cylindrical microdomains under magnetic field has been reported by our group. ${ }^{12}$

To clarify the detailed mechanism of this high orientation of the microcylinders, in this study, we tried

${ }^{\dagger}$ To whom correspondence should be addressed (Tel: +81-3-5734-2633, Fax: +81-3-5734-2888, E-mail: jwatanab@polymer.titech.ac.jp). 
the magnetic alignment of microdomains in various LCBCPs with different molecular weights and components, and in mixtures of LCBCP and LC homopolymer. The results show that a prerequisite for their alignment is the order-disorder transition of the microdomain structure simultaneous with the LC-isotropic liquid transition in the LC segment. When the LCP segment transforms to the nematic phase from the isotropic one, well-defined microcylinders are concurrently formed from weakly segregated state or disordered state, and their alignment is accomplished by the anchoring operation which forces the long axis of the microcylinder lie parallel to the nematic director.

\section{EXPERIMENTAL}

\section{Materials}

The LC homopolymers and the block LC copolymers with PS used in this study,

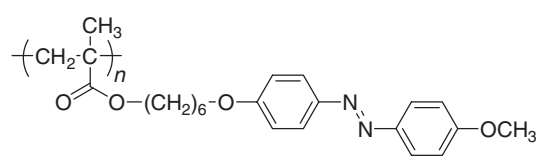

\section{LC homopolymer}

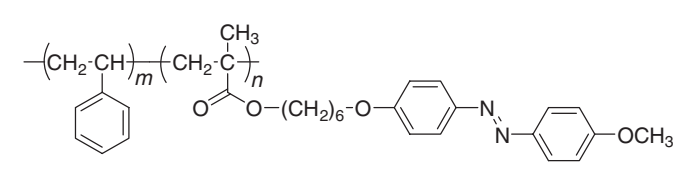

\section{LC block copolymer}

were synthesized by atom transfer radical polymerization. The details about the synthesis are described elsewhere. ${ }^{12}$ These are characterized in Tables I and II, respectively. Here, HP means the LC homopolymer and the succeeding number indicates its number averaged molecular weight, $M_{\mathrm{n}} \times 10^{-2}$. The block co- polymers are designated as $\mathbf{B C P}-\mathbf{m} / \mathbf{n}$ where $\mathbf{m}$ and $\mathbf{n}$ indicate $M_{\mathrm{n}} \times 10^{-2}$ and the weight fraction of the LCP segment (\%), respectively. The mixtures of block copolymer BCP-214/80 and homopolymer HP-130 were also prepared with high LC segment contents from 80 to $100 \%$. These mixtures are designated as Mx where $\mathbf{x}$ is the weight fraction of the LCP segment (\%). Those are listed in Table III.

\section{Measurements}

Thermal behaviors were examined by a PerkinElmer Pyris 1 DSC with a scanning rate of 10 ${ }^{\circ} \mathrm{C} \mathrm{min}^{-1}$ under nitrogen flow. Wide-angle X-ray diffraction (WAXD) patterns were recorded on a flat imaging plate by irradiating Ni-filtered $\mathrm{Cu} \mathrm{K} \alpha$ radiation (Rigaku UltraX18). Small-angle X-ray scattering (SAXS) patterns were measured by a Bruker AXS Nano-STAR.

SR-SAXS measurements for microsegregated structures in the block copolymers and the mixtures were also performed at the BL-10C beam line in a synchrotron radiation facility, Photon Factory (PF) at High Energy Accelerator Research Organization, Tsukuba, Japan and at 4C2 beam line in Pohang Light Source (PLS), Pohang, Korea. The detector equipped at BL-10C in PF was a one dimensional position sen-

Table I. Characterizations of LC homopolymers

\begin{tabular}{lrcl}
\hline Sample & \multicolumn{1}{c}{$M_{\mathrm{n}}{ }^{\mathrm{a})}$} & $M_{\mathrm{w}} / M_{\mathrm{n}}{ }^{\mathrm{a})}$ & Phase behavior $^{\mathrm{b})}\left(\mathrm{Temp} . /{ }^{\circ} \mathrm{C}^{\mathrm{c})}\right)$ \\
\hline HP-92 & 9,200 & 1.15 & $\mathrm{G}(72) \mathrm{SmA}(88) \mathrm{N}(129)$ Iso. \\
HP-130 & 13,000 & 1.16 & $\mathrm{G}(78) \mathrm{SmA}(92) \mathrm{N}(132)$ Iso. \\
HP-191 & 19,100 & 1.27 & $\mathrm{G}(75) \mathrm{SmA}$ (94) N (135) Iso. \\
HP-242 & 24,200 & 1.34 & $\mathrm{G} \mathrm{(77)} \mathrm{SmA} \mathrm{(96)} \mathrm{N} \mathrm{(136)} \mathrm{Iso.}$ \\
\hline
\end{tabular}

a) determined by GPC calibrated with polystyrene standard.

b) G: glassy smectic A, SmA: smectic A, N: nematic, Iso.: isotropic liquid. ${ }^{\mathrm{c})}$ determined by heating DSC measured at a rate of $10^{\circ} \mathrm{C} \mathrm{min}^{-1}$.

Table II. Characterization of LC block copolymers

\begin{tabular}{|c|c|c|c|c|c|c|}
\hline Sample & $M_{\mathrm{n}, \text { total }}{ }^{\text {a) }}$ & $M_{\mathrm{w}} / M_{\mathrm{n}}^{\mathrm{a})}$ & $\begin{array}{l}\Phi_{\mathrm{LC}} \\
/ \%^{\mathrm{b})}\end{array}$ & $\begin{array}{l}\text { Phase behavior }{ }^{\mathrm{c})} \\
\left(\mathrm{Temp} . /{ }^{\circ} \mathrm{C}^{\mathrm{d})}\right)\end{array}$ & $\begin{array}{l}\text { Morphology } \\
\text { in TEM }\end{array}$ & $\begin{array}{l}\text { Magnetic } \\
\text { orientation }\end{array}$ \\
\hline BCP-240/22 & 24,000 & 1.31 & 22 & -e) & - e) & $x$ \\
\hline ВСР-183/43 & 18,300 & 1.34 & 43 & G (93) N (116) Iso. & Lamellar & $\times$ \\
\hline ВCP-418/49 & 41,800 & 1.17 & 49 & $\mathrm{G}\left(-^{\mathrm{e})}\right) \operatorname{SmA}(96) \mathrm{N}$ (134) Iso. & Lamellar & $x$ \\
\hline ВCP-226/51 & 22,600 & 1.46 & 51 & $\mathrm{G}\left({ }^{\mathrm{e}}\right) \operatorname{SmA}(86) \mathrm{N}$ (123) Iso. & Lamellar & $\times$ \\
\hline ВCP-255/61 & 25,500 & 1.19 & 61 & G(70)SmA(93) N (126) Iso. & Lamellar & $x$ \\
\hline ВCP-180/74 & 18,000 & 1.29 & 74 & $\mathrm{G}(55) \operatorname{SmA}(81) \mathrm{N}$ (108) Iso. & Cylinder & 0 \\
\hline ВСР-404/79 & 40,400 & 1.34 & 79 & G(80)SmA (95) N (134) Iso. & Cylinder & $x$ \\
\hline ВCP-214/80 & 21,400 & 1.27 & 80 & G(65)SmA (91) N (122) Iso. & Cylinder & 0 \\
\hline ВCP-261/84 & 26,100 & 1.42 & 84 & $\mathrm{G}\left({ }^{-}{ }^{e}\right) \operatorname{SmA}(90) \mathrm{N}$ (123) Iso. & Cylinder & 0 \\
\hline ВCP-205/87 & 20,500 & 1.32 & 87 & G(70)SmA (95) N (128) Iso. & Cylinder & 0 \\
\hline
\end{tabular}

a) determined by GPC calibrated with polystyrene standard. ${ }^{\text {b) }}$ Weight fraction of PLC segment determined by ${ }^{1} \mathrm{H}$ NMR. ${ }^{c}$ G: glassy smectic A, SmA: smectic A, N: nematic, Iso.: isotropic liquid. ${ }^{\mathrm{d})}$ Determined by heating DSC measured at a rate of $10^{\circ} \mathrm{C} \mathrm{min}^{-1}$. ${ }^{\mathrm{e})}$ Difficult to estimate. 
Table III. Mixtures of BCP-214/80 and H-130

\begin{tabular}{lcc}
\hline \multicolumn{1}{c}{ Sample } & $\begin{array}{c}\text { Weight fraction of } \\
\text { BCP-214/80 }\end{array}$ & $\begin{array}{c}\text { Weight fraction of } \\
\text { LCP segment }\end{array}$ \\
\hline BCP-214/80 & 1.00 & 0.80 \\
M88 & 0.60 & 0.88 \\
M92 & 0.40 & 0.92 \\
M96 & 0.20 & 0.96 \\
M98 & 0.10 & 0.98 \\
HP-130 & 0.00 & 1.00 \\
\hline
\end{tabular}

sitive proportional counter (PSPC) having 512 channels. The scattering profile was obtained by the same manner as described in a previous work. ${ }^{13}$ At PLS, the scattering intensity was measured with a Princeton two-dimensional charge coupled device (CCD) having $2084 \times 2084$ pixels with the size of $57.6 \times 57.6 \mu \mathrm{m}^{2}$. The details on the measurements were described elsewhere. ${ }^{14}$ The one dimensional scattering profile was obtained by reading intensities in the radius direction in the two-dimensional scattering pattern. The numbers of the counter channel or the pixel are connected to the wave vector $q=4 \pi \sin \theta / \lambda$ with using the reflections of the standard materials.

Temperature of the sample was controlled within $1{ }^{\circ} \mathrm{C}$ by using a Mettler FP82 hot stage.

\section{RESULTS AND DISCUSSION}

\section{Transition Behavior of LC Homopolymers and LC} Block Copolymers

HP-130, as a typical example of the LC homopolymers, shows two endothermic peaks at 92 and $132^{\circ} \mathrm{C}$ in the heating DSC thermogram, which are assigned to smectic A (SmA)-nematic (N) and $\mathrm{N}$-isotropic transitions, respectively. On cooling from the SmA phase, crystallization does not take place at all and in place, the glass transition of $\mathrm{SmA}$ is observed at $78^{\circ} \mathrm{C}$. At room temperature, HP-130 necessarily forms the glassy SmA phase. From Table I, we know that the molecular weight affects the transition temperatures, but not the transition behavior. All the homopolymers thus exhibit the glassy SmA, SmA, N and isotropic phases in an order of increasing temperature.

The similar phase transitions are observed for the LCBCPs, BCP-m/n at the temperatures somewhat lower than those of homopolymers (see Table II). The exhibition of clear LC transitions indicates the microphase segregation of LCP segments from PS domains. In fact, transmission electron microscopy (TEM) textures shown representatively in Figure 1 indicate the clear segregation morphology. The type of segregation morphology observed at room temperature by TEM is listed in sixth column of Table II.

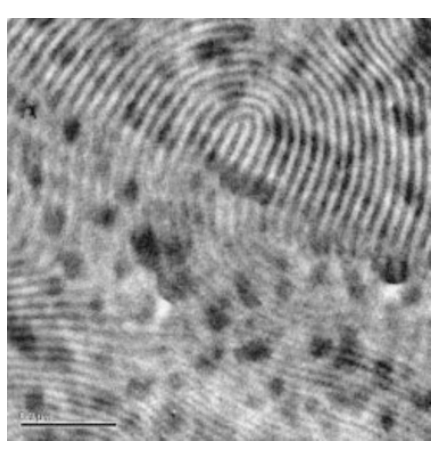

(a) BCP-226/51

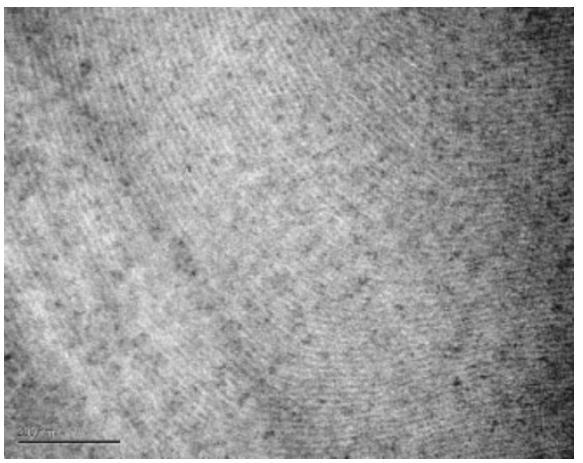

(b) BCP-214/80

Figure 1. Transmission electron microscopic photographs for the ultra-thin section cut out of diblock copolymers: (a) BCP-226/ 51 and (b) BCP-214/80. The bulk samples were annealed for $3 \mathrm{~h}$ at a nematic temperature of $105^{\circ} \mathrm{C}$ and then slowly cooled to room temperature at the rate of $1^{\circ} \mathrm{C} \mathrm{min}^{-1}$. The polystyrene microdomains were stained by $\mathrm{RuO}_{4}$ vapors and thus appear dark.

\section{Magnetic Alignment of Liquid Crystal Mesogens and Microdomains}

The magnetic alignment of a nematic liquid crystal was first examined for the LC homopolymers. There, the molded film sample placed on a Teflon sheet was cooled down from $140^{\circ} \mathrm{C}$ (isotropic phase) to room temperature (glassy SmA phase) at a rate of $10^{\circ} \mathrm{C} \mathrm{min}^{-1}$ in a magnetic field with strength of $5 \mathrm{~T}$ parallel to the film surface.

High orientation has been accomplished for all the homopolymers. The WAXD pattern includes smectic layer reflection and broad outer halo in the direction parallel and perpendicular to the magnetic field, respectively. The latter originates from the liquid-like lateral packing of mesogens within a smectic layer. Accordingly, the mesogens are perfectly aligned parallel to the magnetic field, showing the positive anisotropy of diamagnetic susceptibility, $\Delta \chi=\chi_{\|}-\chi_{\perp}$.

The same procedure was applied for $\mathbf{B C P}-\mathbf{m} / \mathbf{n}$, except that the isotropic melt was annealed at $140^{\circ} \mathrm{C}$ for $10 \mathrm{~min}$ before cooling to diminish the thermal prehistory with respect to the microdomain structure. Figures 2a and 2f show WAXD and SAXS patterns, respectively, observed for the BCP-214/80 with cy- 


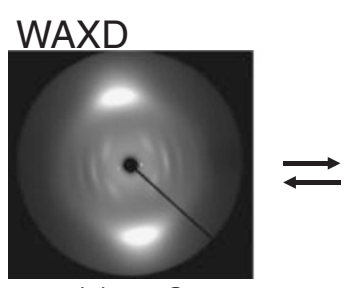

(a) $25^{\circ} \mathrm{C}$

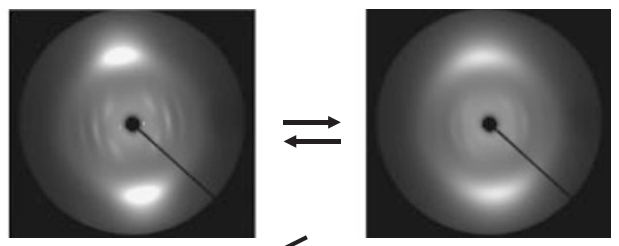

(c) $100^{\circ} \mathrm{C}$

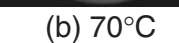

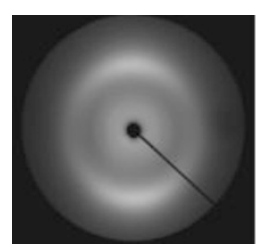

(d) $120^{\circ} \mathrm{C}$
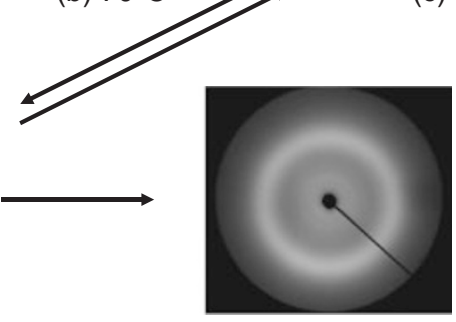

(e) $130^{\circ} \mathrm{C}$

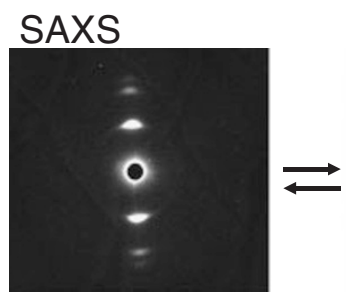

(f) $25^{\circ} \mathrm{C}$

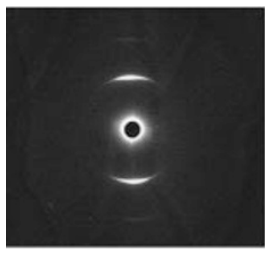

(i) $125^{\circ} \mathrm{C}$

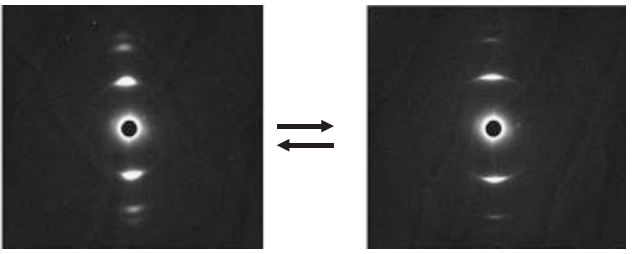

(h) $120^{\circ} \mathrm{C}$

Figure 2. Wide-angle $X$-ray diffraction patterns (a-e) and small-angle $X$-ray scattering patterns ( $f-j)$ measured for magnetically oriented BCP-214/80 at various temperatures. Here, the X-ray beam was irradiated perpendicular to both the film surface and the magnetic field direction. Magnetic field with strength of $5 \mathrm{~T}$ is in horizontal direction. The arrows indicate temperature reversibility of the patterns.

lindrical morphology which was oriented successfully. In the WAXD pattern, the broad reflections appear on meridian similarly as observed in the homopolymers, and in the SAXS pattern, sharp reflections attributable to the lateral packing of microcylinders appear on meridian as well. Thus, the magnetic orientation of the mesophase has induced the orientation of the PS microcylinders with their cylinder axes parallel to the director of mesophase. Inversely, this indicates that the LC mesogens are strongly anchored on the PS microcylinder surface: the LC mesogens tend to align parallel not only to the cylinder surface, but also to the cylinder axis. Such an anchoring effect has been reported. ${ }^{12}$

These high orientations of both the liquid crystal and the microdomain have been accomplished only by cooling the LCBCPs from isotropic liquid temperature to the nematic temperatures at a normal rate of
$10{ }^{\circ} \mathrm{C} \mathrm{min}^{-1}$ in the magnetic field. They do not require the time-consuming procedure adopted in a previous study $^{12}$ where the LCBCPs were cooled from the isotropic temperature at a very slow rate of $0.1{ }^{\circ} \mathrm{C} \mathrm{min}{ }^{-1}$ and then annealed at a nematic temperature for a long time. Important is that the orientation of microdomains is achieved only in the limited copolymers as found in Table II. The lamellar domains in the copolymers having nearly equimolar components are not oriented at all. Among the copolymers having the cylinder domains, only the lower molecular weight ones show the good orientation. The reason will be shown later. It should be noted that in the unoriented microdomains even the mesogenic groups do not align at all. This means that the anchoring effect on the boundary surface of microdomains works on the mesogens' orientation more strongly than the magnetic field. 
Temperature Dependence of WAXD and SAXS Patterns for The Oriented Sample

From Figure 2f, five reflections can be detected with the spacings of $161,93,80,58$ and $54 \AA$. The ratio of their reciprocals is $1: \sqrt{3}: \sqrt{4}: \sqrt{7}: \sqrt{9}$, indicating the hexagonally packed cylinders with a lattice edge of $188 \AA$. The degree of orientation of the microcylinders, $\left\langle P_{2}\right\rangle=\left(3\left\langle\cos ^{2} \beta\right\rangle-1\right) / 2$ was estimated from the intensity distribution $I(\beta)$ of the (100) reflection along the azimuthal angle $(\beta)$, which is taken from the meridian, according to the following equation, ${ }^{15}$

$$
\left\langle\cos ^{2} \beta\right\rangle=\frac{\int_{0}^{\pi / 2} I(\beta) \cos ^{2} \beta|\sin \beta| \mathrm{d} \beta}{\int_{0}^{\pi / 2} I(\beta)|\sin \beta| \mathrm{d} \beta} .
$$

$\left\langle P_{2}\right\rangle$ is $0.97-0.99$, demonstrating the perfect orientation of the microcylinders. This value is interestingly compared with the orientational order parameter of LC mesogens, $S=0.80$, which was estimated from the azimuthal intensity scan of the broad outer halo in Figure 2a by the similar method.

In the SAXS pattern measured by an irradiation along the magnetic field direction, all the reflections appear ring-shaped. This shows the simple planar orientation of the PS cylinders. Thus, it can be concluded that the microcylinders are perfectly aligned parallel to the magnetic field, but there is no preferential orientation of the (100) planes of the hexagonal lattice on the projection perpendicular to the magnetic field direction.

The temperature dependences of the LC orientational order parameter, $S$ and the orientational parameter of microcylinders, $\left\langle P_{2}\right\rangle$ were examined. X-Ray patterns measured at different temperatures are shown in Figure 2 and the determined parameters are plotted against temperature in Figure 3. On heating the sample to any temperature lower than the isotropization temperature of nematic $\mathrm{LC}\left(T_{\mathrm{i}}=122^{\circ} \mathrm{C}\right)$, no distinct change is observed on the SAXS profiles of Figures $2 \mathrm{f}-2 \mathrm{~h}$ and $\left\langle P_{2}\right\rangle$ remains constant around 1 . In contrast, $S$ decreases gradually from 0.8 to 0.4 up to the temperature just below $T_{\mathrm{i}}$ as found in Figure $3 \mathrm{a}$. This reduction in the order parameter is completely recovered on cooling, indicating the conventional temperature dependence of the nematic order parameter. Thus, the high orientation of the cylindrical microdomain structure as well as the uniaxial orientation of the nematic LC is retained if the temperature is set below $T_{\mathrm{i}}$.

On heating to the temperatures above $T_{\mathrm{i}}$, the drastic change took place. The SAXS pattern taken at $135^{\circ} \mathrm{C}$ (Figure $2 \mathrm{j}$ ) shows that the orientation of the microdomain completely disappears. On cooling to the tem- (a)

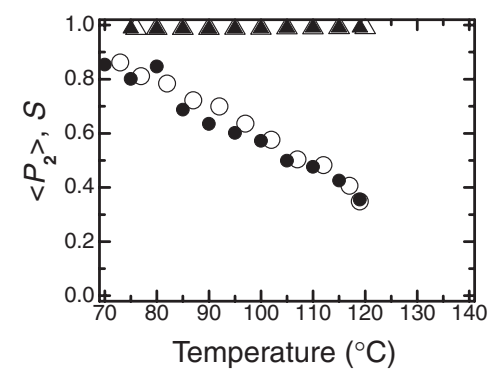

(b)

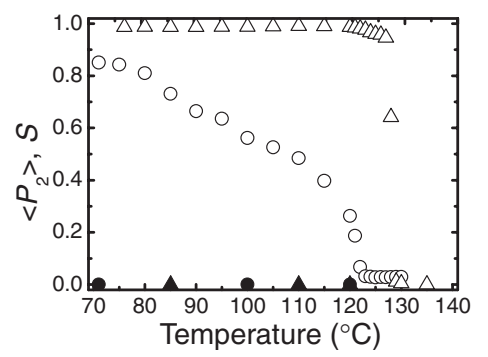

Figure 3. Temperature dependence of the orientational order parameter of liquid crystals $(S)$ (open circle) and the degree of orientation of microcylinder $\left(\left\langle P_{2}\right\rangle\right)$ (open triangle) in the magnetically oriented BCP-214/80 sample on heating to a temperature (a) below $T_{\mathrm{i}}$ and (b) above $T_{\mathrm{i}}$. Closed marks were collected on cooling processes. Once the temperature is raised above $T_{i}$, both parameters irreversibly become zero as indicated by the closed marks as found in (b).

peratures below $T_{\mathrm{i}}$, the orientation of microcylinders did not recover at all and simultaneously the macroscopic uniaxial orientation of the mesophase was lost. This suggests that at $T_{\mathrm{i}}$ the cylindrical microdomains should probably transform to the sphere-like microdomains which are expected from the copolymer component, or to the disordered state. It should be noted that this disappearance of the cylindrical microdomain orientation requires only less than 1 minute, showing that the order-order or order-disorder transformation takes place in very short time span. All other copolymers, BCP-180/74, BCP-261/84 and BCP-205/87, which are magnetically aligned, show the similar behavior.

\section{Orientation Mechanism of Cylindrical Microdomain}

Above results suggest that the prerequisite for microdomain orientation is the concurrent formation of PS cylindrical microdomains on the transformation from the isotropic phase to the nematic phase in the LCP segment. To clarify this point, we examined temperature dependence of the SAXS profiles for the powder samples of BCP-m/n. Typical examples are shown for BCP-418/49, BCP-404/79 and BCP-214/ 80 in Figures 4a, 4b and 5, respectively. BCP-404/79 and BCP-214/80 form the cylindrical microdomain, but the well alignment is accomplished in BCP-214/ 80 but not in BCP-404/79. BCP-418/49 forms the lamellar type of microsegregation which is not aligned 
(a)

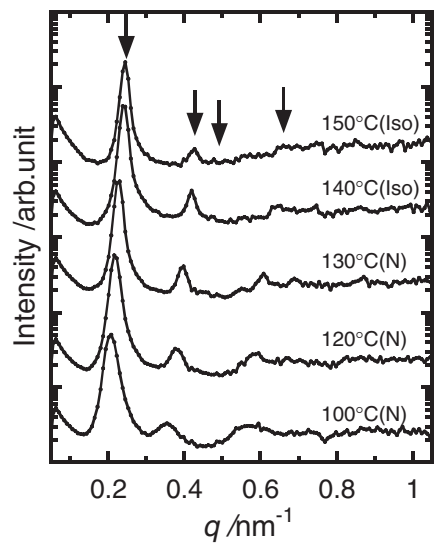

(b)

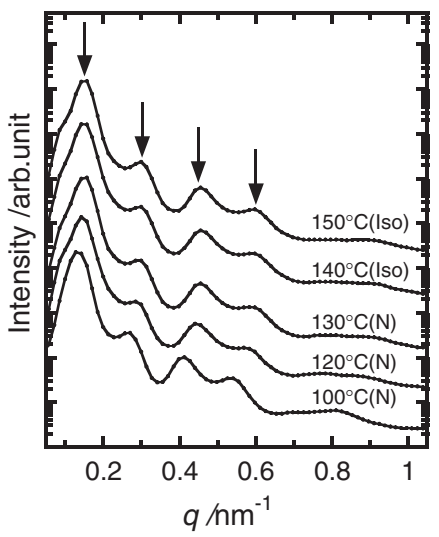

Figure 4. SAXS intensity profiles observed at various temperatures for the powder samples of (a) BCP-404/79 and (b) BCP418/49 whose microsegregation structures were not magnetically oriented. Arrows in (a) and (b) indicate the positions of reflections at $q / q^{*}=1,3^{1 / 2}, 4^{1 / 2}$ and $7^{1 / 2}$ characteristic to hexagonally packed cylinders and that at $q / q^{*}=1,2,3$ and 4 characteristic to lamellar structure, respectively. The measurements were performed at BL-10C in PF.

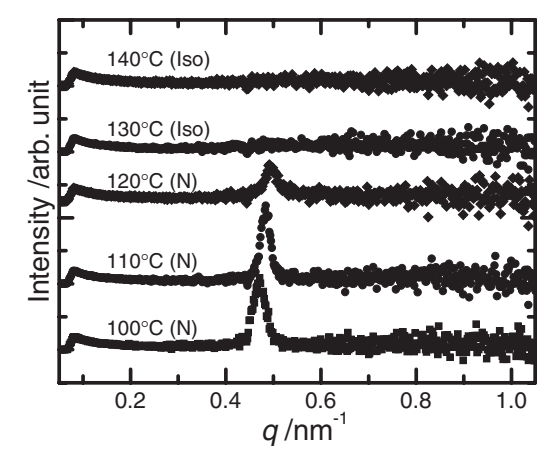

Figure 5. SAXS intensity profiles observed at various temperatures for the powder samples of BCP-214/80 whose microcylinder structure was oriented successfully under the magnetic field. The measurements were performed at $4 \mathrm{C} 2$ beamline in PLS.

at all under the magnetic field.

From Figure $4 \mathrm{a}$, the reflections attributable to the hexagonal packing for the sample BCP-404/79 are maintained up to $150^{\circ} \mathrm{C}$ higher than $T_{\mathrm{i}}\left(134^{\circ} \mathrm{C}\right)$. The maintenance of the lamellar domain in BCP-418/49 is also observed in Figure 4b. In contrast, the reflections attributable to the hexagonal lattice in BCP214/80 disappear at $T_{\mathrm{i}}$ (see Figure 5). In BCP-214/ 80, thus, order-disorder transformation takes place at $T_{\mathrm{i}}$. It should be noticed that such an order-disorder transformation at $T_{\mathrm{i}}$ is commonly observed for all other copolymers which were well oriented under the magnetic field (refer to Table II).

The answer is thus simply given for a question what is the barrier to preparation of the single crystals of LCBCPs by the magnetic field. Let's consider the cases of BCP-418/49 and BCP-404/79. In these cases, no significant variation in size and order takes place on the nematic to isotropic transition. Then, the magnetic alignment of mesogens does not work to align the microdomain because the ordered microdomains with a long correlation length are hardly rearranged. On the contrary, in all the copolymers will oriented under the magnetic field, the ordered cylindrical microdomain is concurrently formed from the weakly segregating or disordered state on the isotropic-nematic transformation of LCP segments. The cylindrical microdomains would be formed under the magnetically aligned nematic LC field, and their orientation would be accomplished by the interplay between the orientations of the liquid crystal and microsegregation structures (so called anchoring operation). ${ }^{11}$ Anchoring of the mesogens on the microcylinder surface induces the long axis of the microcylinders to prefer to align along the nematic director. The perfect alignment of LCBCP microdomains by magnetic field thus requires both the anchoring operation on the mesogens in the interface of microdomains and the exhibition of disorder-order transition on the isotropic-nematic transition of the LCP segments.

Unordinary Microsegregation Morphology in Liquid Crystalline Field as Detected in Magnetically Aligned Samples

Interesting here is that the PS segment in BCP-214/ 80 forms cylinder microdomain in the LC matrix regardless of its low weight fraction of $20 \%$ which indicates the PS segment usually forms sphere microdomain. With how low content can the PS segment maintain the cylinder microdomain in the anisotropic LC matrix? This can be examined by the magnetic orientation of the mixtures of block copolymer BCP214/80 and LC homopolymer HP-130 with low PS contents of $12-2 \%$ (see Table III). All the mixtures show clear oriented SAXS patterns (see Figure 6) as well as the oriented WAXD patterns, however, the higher ordered reflections characteristic to the hexagonal packing are lost for the mixtures with the PS contents of $8-2 \%$. The degree of cylinder orientation, 


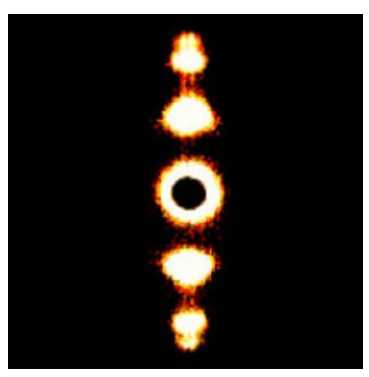

(a) M88

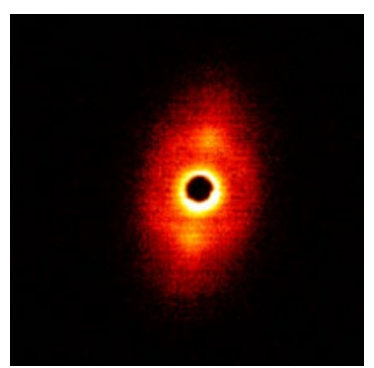

(c) M96

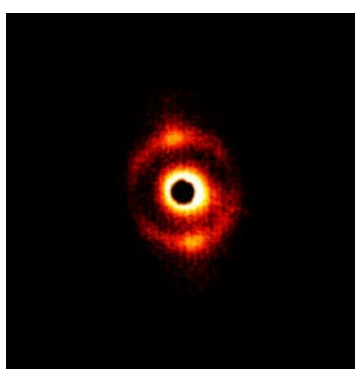

(b) M92

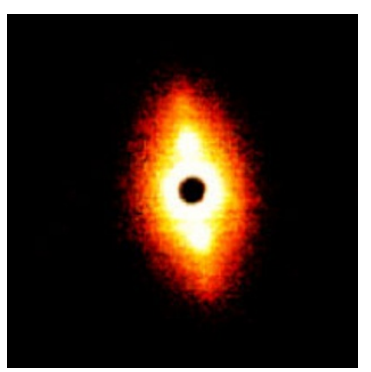

(d) M98
Figure 6. SAXS patterns for the magnetically oriented mixtures of a block copolymer BCP-214/80 and a LC homopolymer HP-130. Magnetic field with strength of $5 \mathrm{~T}$ is in horizontal direction.

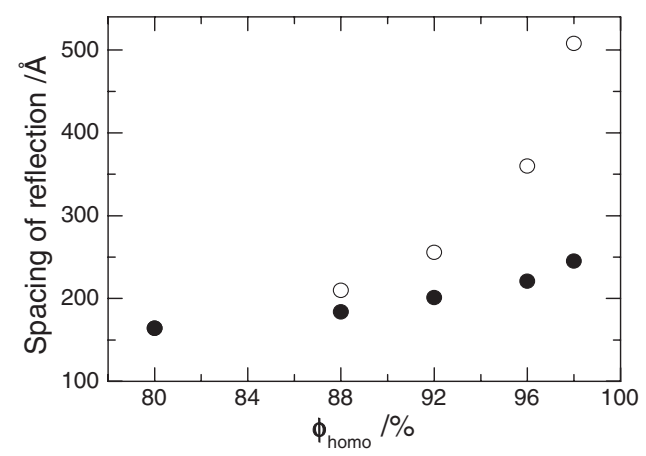

Figure 7. Variation of the inner reflection spacing determined from the SAXS patterns (closed circle) on the LCP segment content for BCP-214/80, M88, M92, M96, and M98. The open circle indicates the expected spacing if the homopolymer HP-130 dilutes homogeneously the LCP matrix region of block copolymer BCP-214/80 with cylindrical microdomain.

$\left\langle P_{2}\right\rangle$ also decreases with increasing the LC content while the LC orientational order parameter, $S$, is nearly constant around 0.8 . The values of $\left\langle P_{2}\right\rangle$ estimated for M88, M92, M96 and M98 are 0.98, 0.80, 0.70 and 0.66 , respectively. In Figure 7 , the spacing of the innermost reflection in the SAXS pattern is plotted against the LCP content by closed circle. The spacing increases steadily with increase in the LCP content. By assuming the uniform dilution of the LC homopolymer into the LCP matrix part of cylindrical microdomain, the expected spacing can be calculated with the densities of the LCP and PS domains of
1.30 and $1.05 \mathrm{~g} \mathrm{~cm}^{-3}$, respectively. The calculated spacing is plotted by open circle in Figure 7. The observed spacing roughly corresponds to the calculated one for M88 while a significant deviation is observed in the mixtures with the higher LCP contents of 92$98 \%$. This deviation may be due to the inhomogeneous dilution. Irrespective of this fact, the highly oriented SAXS pattern dictates that the cylindrical or anisotropic rod-like segregation is still maintained up to the extremely low PS content of $2 \%$. Such a persistent formation of cylindrical or anisotropic microdomain is reasonable in the LC field since the unidirectional LC field is inconsistent with the sphere-like segregation which results in significant defects such as line and point disclinations in the LC phase. Finally, it should be noted that all these oriented patterns are lost at temperature near $T_{\mathrm{i}}$ of LCP segment as observed in BCP-214/80.

\section{CONCLUSION}

By taking advantage of the magnetic orientation of nematic liquid crystal, we have achieved the well orientation of the microdomains in the LC block copolymers. Well-aligned samples have been prepared only in the low molecular weight copolymers with PS cylindrical microdomains dispersed in a LCP matrix, in which the cylindrical microdomain structure is disordered simultaneously with the transformation of the nematic phase of the LCP segment into isotopic liquid phase. The alignment has been accomplished with both the axis of microcylinder and the $\mathrm{n}$-director of the nematic LC parallel to the magnetic field direction. It is thus concluded that the prerequisites for successful magnetic alignment of microdomain structure are the exhibition of disorder-order transition at the isotropic to nematic transition of LCP segment and the anchoring effect in the interface of microdomains.

Well-oriented cylindrical or anisotropic microsegregated structures are observed for the mixtures of a LCBCP and a LC homopolymer even with the extremely low content of PS segments of $12-2 \%$. Such a persistent formation of anisotropic microsegregated structures is forced by the anisotropic field of liquid crystalline matrix. In other words, the cylinder-type of microsegregation is more energetically favored than the sphere-type one in LCBCPs since the liquid crystal monodomain without defects such as line and point disclinations can be achieved by the anisotropic rod-like segregation, but not by the sphere-like one.

Acknowledgment. The synchrotron radiation Xray measurements at Pohang Light Source (PLS) were supported by MOST and POSCO. 


\section{REFERENCES}

1. P. Davidson, L. Noirez, J. P. Cotton, and P. Keller, Liq. Cryst., 10, 111 (1991).

2. L. Noirez, P. Davidson, W. Scwarz, and G. Pépy, Liq. Cryst., 16, 1081 (1994).

3. L. Noirez, P. Keller, and J. P. Cotton, Liq. Cryst., 18, 129 (1995).

4. M. Yamada, T. Iguchi, A. Hirao, S. Nakahama, and J. Watanabe, Macromolecules, 28, 50 (1995).

5. M. Yamada, T. Itoh, R. Nakagawa, A. Hirao, S. Nakahama, and J. Watanabe, Macromolecules, 32, 282 (1999).

6. T. Itoh, N. Tomikawa, M. Yamada, and J. Watanabe, Polym. J., 33, 783 (2001).

7. H. Fischer, S. Poser, and M. Arnold, Liq. Cryst., 18, 503 (1995).

8. G. Mao, J. Wang, S. R. Clingman, C. K. Ober, J. T. Chen, and E. L. Thomas, Macromolecules, 30, 2556 (1997).
9. J. Adams and W. Gronski, in "Liquid Crsytal Polymers," D. A. Wein and C. K. Ober, Ed., American Chemical Society, Washington DC, 1990.

10. I. W. Hamley, V. Castelletto, Z. B. Lu, C. T. Imrie, T. Itoh, and A. Al-Hussein, Macromolecules, 37, 4798 (2004).

11. C. Osuji, P. J. Ferreira, G. Mao, C. K. Ober, J. B. Vander Sande, and E. L. Thomas, Macromolecules, 37, 9903 (2004).

12. N. Tomikawa, Z. Lu, T. Itoh, C. T. Imrie, M. Adachi, M. Tokita, and J. Watanabe, Jpn. J. Appl. Phys., Part 2, 44, L711 (2005).

13. M. Tokita, K. Tokunaga, S. Funaoka, K. Osada, and J. Watanabe, Macromolecules, 37, 2527 (2004).

14. S. Kang, M. Tokita, K. Ogino, T. Doi, T. Takahashi, H. Takezoe, and J. Watanabe, Phys. Rev. E: Stat., Nonlinear, Soft Matter Phys., 73, 011701 (2006).

15. R.-J. Roe, "Methods of X-ray and Neutron Scattering in Polymer Science," Oxford University Press, New York, 2000. 